\title{
Editorial principles
}

Lecture notes taken during Alfred North Whitehead's classes were recorded not only by his students but also by recent graduates and both junior and senior faculty colleagues. Some sets of notes were deposited in various library archives with which their owners were affiliated, while others were privately retained and discovered later by Whitehead's biographer, Victor Lowe, or by members of the Whitehead Research Project. ${ }^{1}$

In editing lecture notes taken during Whitehead's classes, the editors have operated on a policy of minimal interference with the text. Although we are dealing for the most part with notes on Whitehead's lectures taken by others, the text is the closest thing we have to Whitehead's words. This being the case, we correct what is clearly wrong (such as typographical errors and incorrect bibliographic information) but make no attempt to edit the text aggressively.

The following principles, listed alphabetically, have governed the way in which the text has been edited, standardised or silently corrected, across the Critical Edition. Elaborations and additions specific to each volume may be made as a matter of editorial discretion.

- Angle brackets. Angle brackets ( $($ and $\rangle)$ have been used to indicate editorial intervention, since both parentheses and square brackets have been used by the original note-takers.

- Capitalisation. Capitalisation has been standardised without record according to the sixteenth edition of the Chicago Manual of Style.

- Contractions/shorthand.

- If the shorthand or contraction is unambiguous, it has been silently expanded without record. This includes ditto marks.

1. For reflections on Whitehead's teaching style, see: A. H. Johnson, Whitehead and His Philosophy (Lanham: University Press of America, 1983) and 'Whitehead as teacher and philosopher', Philosophy and Phenomenological Research, vol. 29(3) (I969), pp. 35I-76; William Ernest Hocking, 'Whitehead as I knew him', in George L. Kline (ed.), Alfred North Whitehead: Essays on His Philosophy (Englewood Cliffs: Prentice Hall, 1963), pp. 7-17 ; 'A. N. Whitehead in Cambridge, Mass.', which is chapter 5 of Dorothy Emmet's Philosophers and Friends: Reminiscences of Seventy Years in Philosophy (London: Macmillan, 1996); Victor Lowe's biography of Whitehead, Alfred North Whitehead: The Man and His Work, Volume I and Volume 2 (Baltimore: Johns Hopkins University Press, 1985, 1990); Lester S. King's letter to Lewis Ford, 3 May 1978 (LET322), in the Whitehead Research Project Archives, Center for Process Studies, Claremont School of Theology, Claremont, California, USA; also Lawrence Bragg's letter to Victor Lowe, 8 May 1969, in the papers of William Henry Bragg, Box 37, Folder B, Item 44, Royal Institution of Great Britain, London, UK; Harvey H. Potthoff's diary entry for 2r May 1936, diary held in the Harvey H. Potthoff Papers (Iliff 3-F), Series VII, Sub-Series G, Iliff School of Theology Archives, Denver, Colorado, USA; and George P. Conger's diary entry 'Notes on Whitehead's class lectures at Harvard University, 1926-1927', in Box 4 of the George P. Conger Papers (Mss 20), Literary Manuscripts Collection, University of Minnesota Libraries, Minneapolis, USA (that diary is to be published in a further volume of this Critical Edition); see also the Introduction to the present volume. 
- If a contraction or word(s) seems legible, but its meaning is ambiguous or unclear, a footnote has been added explaining the difficulty.

- If the editors have a strong guess but cannot be sure about the reading of a word or a portion of a word, then they have placed angle brackets around those markings with a question mark at the end (e.g. (example?)); or a footnote has been added.

- If a word can be supplied through context, but is otherwise illegible, it has been placed within angle brackets with a question mark (e.g. 'Each age has its (own?) philosophy'); or a footnote has been added.

- Dashes. Dashes have all been standardised as en dashes.

- Deletions. Legible deletions have been retained and marked with strikethroughs (deletion). Insignificant accidental deletions have been silently removed without record. Insignificant accidental deletions include misspellings (freind friend) and false starts (toom much).

- Diagrams/figures. Whenever possible, diagrams and figures that would have appeared on the blackboard (and therefore were drawn by Whitehead) have been faithfully recreated or scanned and inserted in their original location relative to the rest of text.

- Doodles. Insignificant doodles have not been reproduced, but footnotes will indicate their existence.

- Illegible words. If a single word is completely illegible, the editors have

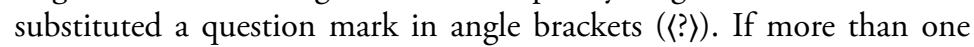
word is illegible, the editors have used an ellipsis or series of ellipses of the approximate length of the illegible text within angle brackets and a question mark at the end $(\langle\ldots \ldots .\rangle$.$) ; or a footnote has been added.$

- Interlineations. Authorial interlineations have been placed in their intended locations and marked with carets. Carets pointing upward $(\wedge$ interlineation $\wedge)$ indicate that the marked word has been moved to its present location from below, while carets pointing downward (Vinterlineationv) indicate that the marked word has been moved to its present location from above.

- Italics. All book titles and foreign words have been italicised. If a book title is underlined or enclosed in quotation marks, this has been silently removed and italics used instead.

- Line and page breaks. The finished editorial text will not retain the author's original line or page breaks, in most cases, and will not generally mark where line breaks occur, but for page breaks a record will be inserted between upright bars (|example|).

- Line spacing. Unusually large or small amounts of space between lines or paragraphs have not been retained in the finished text, in most cases, and no record has been made of them; but where deemed important to understanding the note-taker's intention some spacing may have been retained or a note added.

- Marginal text. Text sometimes appears in odd places on the page (e.g. vertical text, or text that has been circled and placed in the corner of the 
page or in the margin). Placement of such text is a matter of editorial discretion, on a case-by-case basis. A footnote has been added explaining the original positioning of any text whose intended location within the larger text is ambiguous (e.g. 'This line originally appeared in the upper-left corner of the page').

- Markings. The retention of other marginal marks for emphasis, such as vertical lines, braces, arrows, or check marks, has been a matter of editorial discretion. See also 'Underlining'.

- Markings not made by original author. Any markings not made by the original author have been removed without record. These include archival markings, or any markings made by previous transcribers. If ambiguous, a footnote has been added.

- Misspellings. Misspellings have been corrected without record.

- Notes. The footnotes are of two types.

- Editorial notes/textual difficulties. These notes explain textual treatment (e.g. difficulties with textual interpretation or describing odd text positioning).

- Substantive notes/contextual information. These provide further information and context for the reader (e.g. information on a person or book that Whitehead mentions).

- Page headers (including dates). The note-takers' headers have been standardised to a uniform format. Dates marked on the second page or with page numbers may be retained at the editors' discretion if there are no other indicators of page breaks.

- Paragraph indentation. The indentation for paragraphs has been made uniform. However, for outline-style notes with a clear hierarchy the different levels of indentation have sometimes been preserved as a matter of editorial discretion.

- Punctuation. Punctuation may be changed without record for purposes of clarity; however, editors have been extremely conservative when making such changes, especially in relation to the addition of punctuation where the lecture notes are attempting to capture an oral presentation.

- Separator lines. Lines or other marks that the author has used to clearly separate one piece of text from another may be retained or silently removed as a matter of editorial discretion.

- Supply, editorial. Editors have inserted missing articles, prepositions or

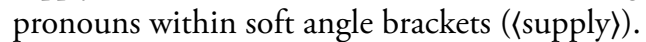

- Underlining. Underlining (including double and wavy underlines) for emphasis has been retained, with the exception of underlines clearly not made by the original author and underlines of book titles, which have been italicised instead; the editors have not added underlining. 